\title{
Grasp synthesis from example: tuning the example to a task or object
}

\author{
Nancy S. Pollard and Alon Wolf \\ Carnegie Mellon University nsp@cs.cmu.edu | alon.wolf@cmu.edu
}

Summary. Many approaches to grasp synthesis do not scale well as the desired number of contacts is increased. In previous work [21], we have presented a technique that makes use of an example grasp to make synthesis tractable for grasps having larger numbers of contacts. However, an interesting side effect of this approach is that results can pick up quirks that are present in the example, some of which may be undesirable. We are beginning to explore techniques to tune an example grasp for a specific collection of task wrenches and to a given object geometry. This paper presents preliminary results.

\section{Introduction}

Many natural grasps incorporate large areas of contact between the hand and the surface of the grasped object (Figure 1). Representing these contact areas using local contact models would require a relatively large number of contacts per grasp. In previous work [21], we have demonstrated a technique for synthesizing grasps from examples that is efficient for grasps having a large number of contacts, and Figure 2 shows some of these results. Independent contact regions are constructed so that one contact in each region guarantees the grasp will preserve desired properties of the example. We have used this algorithm to geometrically construct families of grasps that are force-closure, partial force-closure, and that meet or exceed quality thresholds for metrics reflecting the ability to efficiently apply specific task wrenches to the grasped object.

One difficulty with constructing a solution around an example grasp, however, is that the results tend to preserve quirks such as incidental asymmetries that are present in the example. Consider Figure 3. The complete force / torque space for this $2 \mathrm{D}$ example is three-dimensional: $\left(f_{x}, f_{y}, \tau\right)$. Suppose that the task involves primarily forces in the vertical direction $\left(f_{y}\right)$ and torques applied about the object center of mass $(\tau)$. An example with asymmetries will result in contact regions with asymmetries, as shown in the left hand figure, 

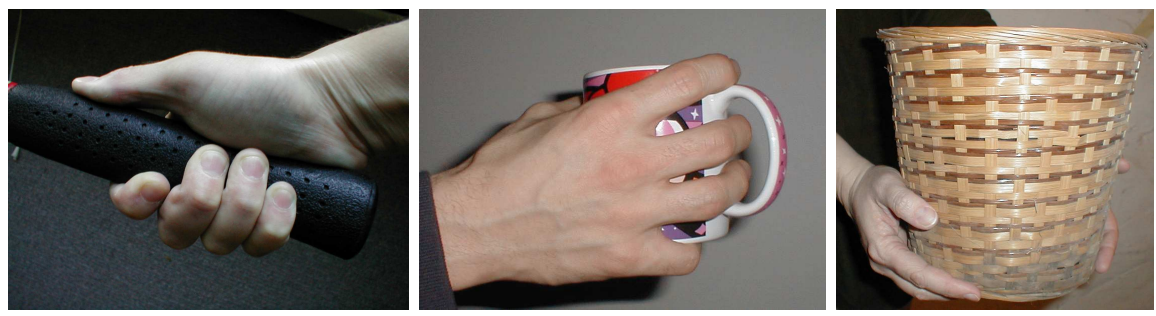

Fig. 1. Many grasps take advantage of extended areas of contact between the hand and object. Abstracting these grasps using local contact models would require a large number of contacts.

while a more symmetrical example will produce a larger and more intuitive set of contact regions for this symmetric object.

This paper briefly reviews our approach for synthesizing grasps from examples (Section 3), describes why this problem occurs, and suggests two ways to adjust examples by tuning them to a given task and to the geometry of a particular object (Sections 4 and 5).

\section{Background}

We assume that the goal of grasp synthesis is to select a good set of contact points on the surface of an object, given knowledge of that object's geometry and a quality metric.

Algorithms for grasp synthesis can be divided into optimization approaches (e.g., $[10,15,17,14,11,30,31])$ and constructive techniques (e.g., $[19,18])$. Constructive techniques are also frequently used in non-prehensile manipulation (e.g., $[16,20,27,6,7,1,13,29])$.

In work closest to ours Ponce and his colleagues [23, 22, 24] and Chen and Burdick [4] describe algorithms for optimizing the sizes of independent contact regions for two-to-four-fingered grasps. In other related work, van der Stappen et al. [28], Liu [12], and Li et al. [9] describe a variety of techniques to compute all force closure grasps for 2D grasps. Optimal independent contact regions could be extracted from the results of their algorithms using the approach described in Ponce et al. [24]. None of these approaches have been extended beyond 4 contacts.

Most previous work in grasp synthesis has focused on a very small, typically minimal number of contacts. A variety of efficient grasp synthesis techniques are available when the number of contact points is small. However, if a grasp having a large number of contact points is desired, it is not clear how to extend these techniques in a tractable manner, with the exception of local numerical optimization (e.g., [31]). When grasps have large numbers of contacts, local optimization may have difficulties with local minima. In addition, 

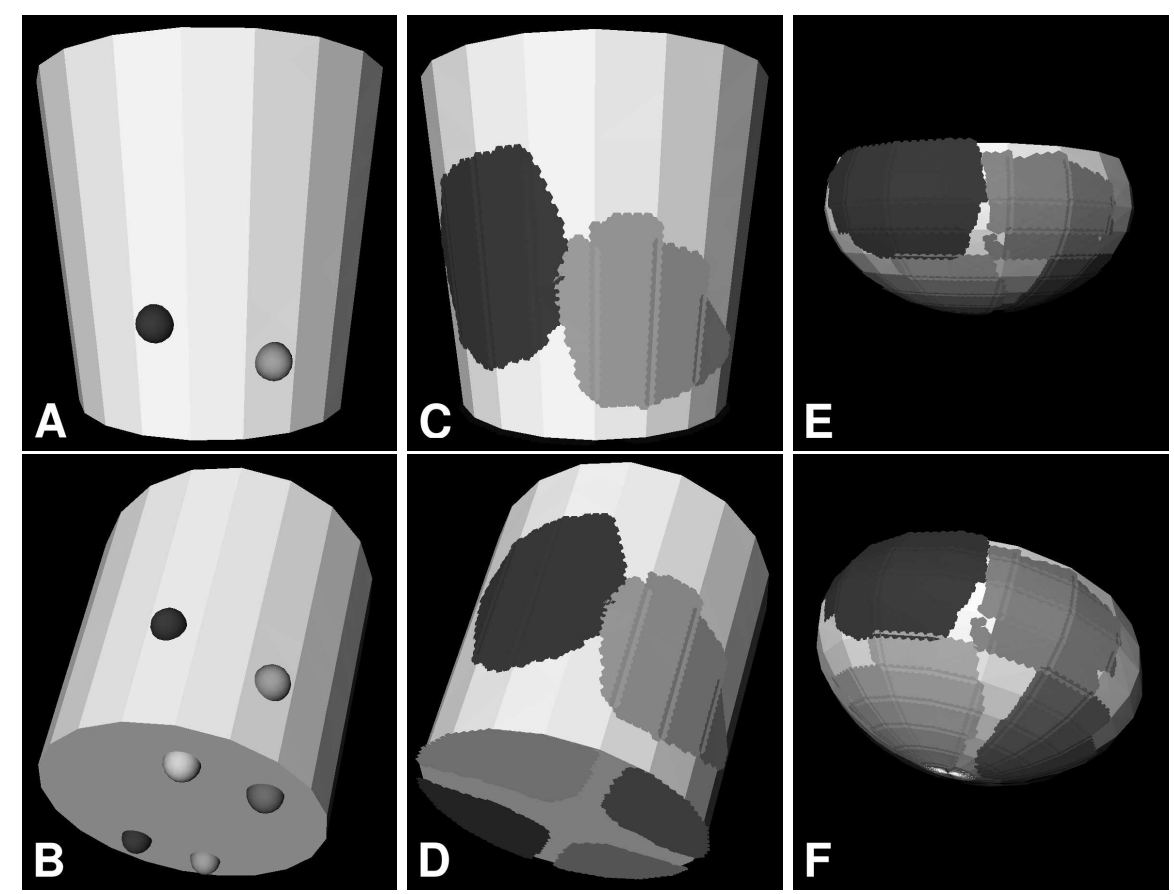

Fig. 2. Our algorithm converts an example grasp into an equivalence class of grasps that can be projected onto any object geometry. One contact in each region guarantees a grasp that preserves desired properties of the example grasp. (A,B) An example with eight hard-finger contacts and a friction coefficient of 1.0. (Contacts on the opposite side mirror those shown.) (C,D) Results for the basket based on this example. The task is to support the object against gravity, and the resulting grasps are "90\% as good as the example." In this example, the metric of Zhu, Ding, and $\mathrm{Li}[30]$ is used to define a high quality grasp as one where a given task can be performed without excessive contact forces. (E,F) Results for the bowl, based on the same example and quality requirement.

when objects have many faces to be considered (e.g. the bowl in Figure 2), any algorithm that must consider all combinations of faces will be prohibitively expensive.

Constructive approaches have the conceptual advantage over numerical optimization techniques that they may be less prone to local minima. However, we believe at this point that the user must provide some additional input information to extend constructive approaches to a large number of contacts while also treating all contacts in an equivalent manner.

By constructing results around an example grasp, we can to some extent achieve this goal. Our paper builds on the ideas of Ponce et al. [24] and others to synthesize independent contact regions. In contrast to previous work, how- 

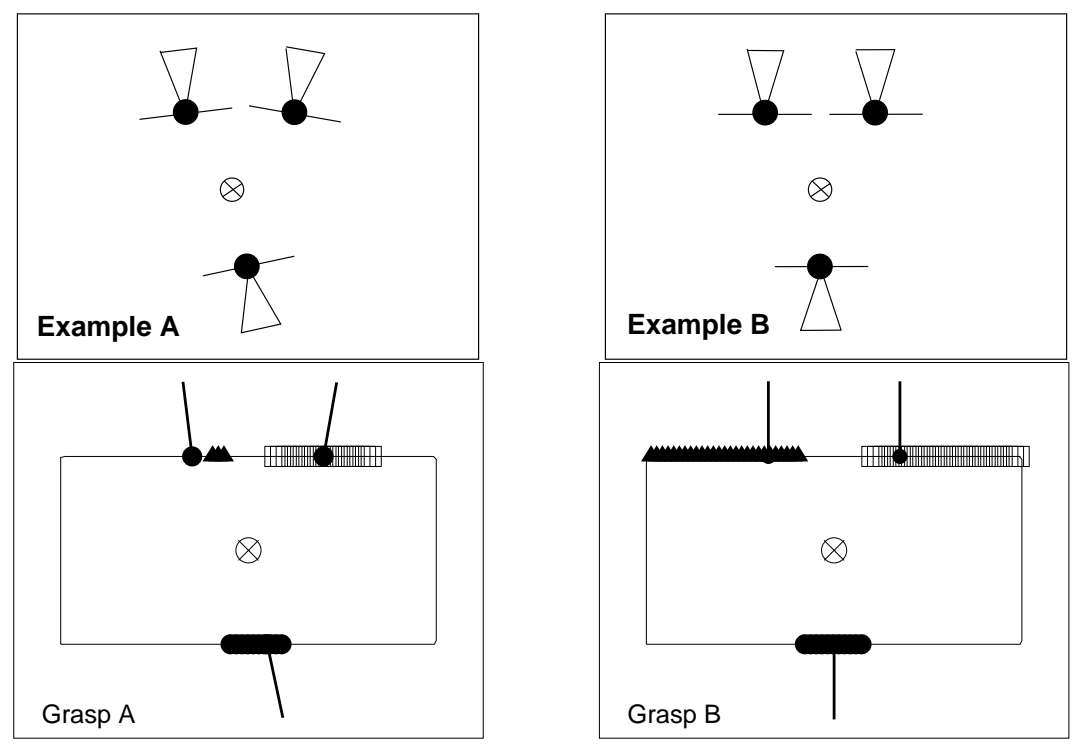

Fig. 3. (Left) An example with asymmetries produces contact regions that also have asymmetries. (Right) A symmetric example for comparison. The quality of grasps represented in these figures has the same lower bound in both cases.

ever, we make use of an example grasp and construct results that preserve closure properties of that example. This use of an example makes a polynomial time algorithm possible. Our grasps are not in general optimal, but they can be constructed to meet user-specified quality bounds. Our approach was presented previously in [21], and this paper describes one way to improve those results by making adjustments to the original example.

Two issues ignored in the grasp synthesis problem assumed in this paper are second-order effects and the kinematics of the mechanism that will grasp the object. Second-order analysis can provide insight into the stability of the grasp with respect to external perturbations (e.g. [25, 26]). The kinematics of the mechanism actually determine whether force closure and other properties of a grasp are achievable [2].

An overview of grasping research, including work in grasp synthesis, can be found in Bicchi [3].

\section{Grasp Synthesis from Example}

The goal of our grasp synthesis algorithm is to create a family of grasps such that all grasps in the family have certain properties, which are specified at design time by the user. The algorithm we use is deterministic (i.e., does not 


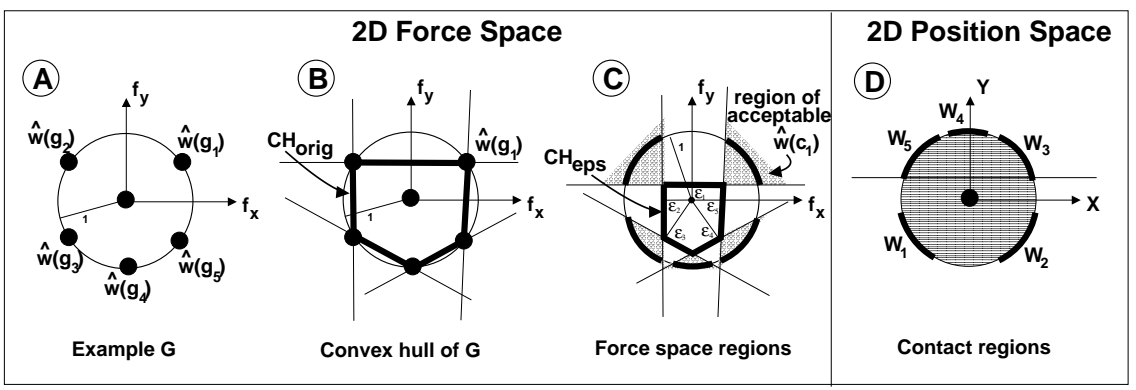

Fig. 4. $2 \mathrm{D}$ example construction of regions $W_{n}(G, \epsilon)$. (A) A frictionless example grasp $\mathrm{G}$ consisting of five contact wrenches. (B) $\mathrm{CH}_{\text {orig }}$ is the convex hull of these contact wrenches. Halfspace boundaries are also shown. (C) $\mathrm{CH}_{\text {eps }}$ is constructed from $\mathrm{CH}_{\text {orig }}$ by moving each halfspace boundary $h$ to distance $\epsilon_{h}$ from the origin. The region of acceptable wrenches $\hat{\mathbf{w}}\left(c_{n}\right)$ is an intersection of exterior halfspaces of $\mathrm{CH}_{\text {eps }}$. (D) Each such region can be mapped to a set of contact points on an object (Section 3.3) to obtain target contact regions $W_{n}(G, \epsilon)$. Grasps in the family $W(G, \epsilon)$ have one contact in each of these regions.

involve search) and it involves constructing this family around a given example grasp.

Figure 4 provides a 2D illustration of the construction process. Suppose we are given an example grasp $G$ having $N$ contacts $g_{1}, \ldots, g_{N}$. Wrenches available at each contact are represented as a linear combination of $L$ extremes. For example, a hard finger contact with friction may be represented using $L$ samples bounding the friction cone at that contact point. The example, then, can be represented as the collection of $N L$ extreme wrenches $\hat{\mathbf{w}}_{l}\left(g_{n}\right)$.

$$
G=\left\{\hat{\mathbf{w}}_{1}\left(g_{1}\right), \ldots, \hat{\mathbf{w}}_{L}\left(g_{N}\right)\right\}
$$

We assume that the $\hat{\mathbf{w}}_{l}\left(g_{n}\right)$ span $\mathbb{R}^{6}$, although they may not positively span $\mathbb{R}^{6}$.

The construction process begins with a volume in $\mathbb{R}^{6}$, the convex hull of all of the $\hat{\mathbf{w}}_{l}\left(g_{n}\right)$. This volume is significant because it captures the closure properties of $G$ and is a first step in constructing many wrench-based quality measures. We will represent this volume, $\mathrm{CH}_{\text {orig }}$, as a collection of halfspaces, each expressed as an outward pointing normal $\hat{\mathbf{n}}_{h}$ and a distance from the wrench space origin $d_{h}$.

$$
C H_{\text {orig }}(G)=\left\{\left[\begin{array}{c}
\hat{\mathbf{n}}_{1} \\
d_{1}
\end{array}\right],\left[\begin{array}{c}
\hat{\mathbf{n}}_{2} \\
d_{2}
\end{array}\right], \ldots,\left[\begin{array}{c}
\hat{\mathbf{n}}_{H} \\
d_{H}
\end{array}\right]\right\}=\operatorname{ConvexHull}(G)
$$

To represent the connection between halfspace boundaries in $C H_{\text {orig }}$ and wrenches $\hat{\mathbf{w}}_{l}\left(g_{n}\right)$, we define index set $\rho_{n, l}$ so that $h \in \rho_{n, l}$ implies that halfspace boundary $h$ passes through point $\hat{\mathbf{w}}_{l}\left(g_{n}\right)$. 


$$
\rho_{n, l}(G)=\left\{h:\left(\hat{\mathbf{w}}_{l}\left(g_{n}\right) \cdot \hat{\mathbf{n}}_{h}\right)=d_{h}\right\}
$$

For each halfspace $h$, we choose some $\epsilon_{h}$ to reflect requirements of the task. Choosing $\epsilon_{h}=d_{h}$ will match capabilities of the example grasp, but if the example is unnecessarily strong in certain directions, setting $\epsilon_{h}$ to a value less than $d_{h}$ will allow greater flexibility in contact placement. Details on setting $\epsilon_{h}$ can be found in Section 3.2.

Let $\epsilon=\left[\epsilon_{1} \ldots \epsilon_{H}\right]$. Based on $C H_{\text {orig }}(G), \rho_{n, l}(G)$, and task variables $\epsilon$, we define an equivalence class of grasps $W(G, \epsilon)$ as a set of regions $W_{n}(G, \epsilon)$, one for each contact, as follows:

$$
\begin{gathered}
W(G, \epsilon)=\left\{c_{1}, \ldots, c_{N}: c_{n} \in W_{n}(G, \epsilon), n=1, \ldots, N\right\} \\
W_{n}(G, \epsilon)=\bigcap_{l=1}^{L} W_{n, l}(G, \epsilon)
\end{gathered}
$$

$W_{n, l}(G, \epsilon)=\left\{c_{n}: \exists \alpha_{l}\right.$ s.t. $\left.\left(\left(Y\left(c_{n}\right) \alpha_{l}\right) \cdot \hat{\mathbf{n}}_{h} \geq \epsilon_{h}\right) \forall h \in \rho_{n, l}, \quad \alpha_{l} \geq \mathbf{0}, \quad\left\|\alpha_{l}\right\|_{L_{1}}=1\right\}$

where $Y\left(c_{n}\right)$ is the $6 x L$ matrix of extreme wrenches in the contact model for contact $c_{n}$.

$$
Y\left(c_{n}\right)=\left[\begin{array}{llll}
\hat{\mathbf{w}}_{1}\left(c_{n}\right) & \hat{\mathbf{w}}_{2}\left(c_{n}\right) & \ldots & \hat{\mathbf{w}}_{L}\left(c_{n}\right)
\end{array}\right]
$$

For any grasp in the set $W$, contact $c_{n}$ is meant to correspond directly to the contact $g_{n}$ in $G$. In other words, the role of contact $c_{n}$ is defined by the set of wrenches $\hat{\mathbf{w}}_{1}\left(g_{n}\right), \ldots, \hat{\mathbf{w}}_{L}\left(g_{n}\right)$ from the example grasp. The valid region for contact $c_{n}$ is constructed as the intersection of $L$ regions $W_{n, l}$, one for each wrench extreme $\hat{\mathbf{w}}_{l}\left(g_{n}\right), l=1, \ldots, L$. Region $W_{n, l}$ is based on the intersection of exterior halfspaces associated with $\hat{\mathbf{w}}_{l}\left(g_{n}\right)$ (i.e., halfspaces indexed by $\rho_{n, l}$ ) after those halfspaces have been adjusted along their normals to distances $\epsilon_{h}$ : some unit wrench available at contact $c_{n}$ must fall within this intersection. Sections 3.1 and 3.2 show how this particular definition makes it possible to control closure and quality properties of all grasps in $W(G, \epsilon)$.

In the frictionless case, $L=1$ and subscript $l$ is not needed, resulting in the following expression for contact region $W_{n}$, which is much cleaner and is illustrated in Figure 4D.

$$
W_{n}(G, \epsilon)=\left\{c_{n}: \hat{\mathbf{w}}\left(c_{n}\right) \cdot \hat{\mathbf{n}}_{h} \geq \epsilon_{h} \forall h \in \rho_{n}\right\} \quad \text { (frictionless case only) }
$$

\subsection{Grasp Properties}

Given this construction technique, what can we say about grasps in $W$ ? In [21], we show the following:

Proposition 1. Suppose we are given grasp $C$ having contacts $\left\{c_{1}, \ldots, c_{N}\right\}$ and grasp family $W(G, \epsilon)$ constructed as in Equation 4.

From Equation 2, $C H_{\text {orig }}(G)$ is the convex hull of the unit wrench extremes of $G$ : 
$C H_{\text {orig }}(G)=$ ConvexHull $\left\{\hat{\mathbf{w}}_{1}\left(g_{1}\right), \ldots, \hat{\mathbf{w}}_{L}\left(g_{N}\right)\right\}=\left(\left[\begin{array}{ll}\hat{\mathbf{n}}_{1} & d_{1}\end{array}\right]^{T}, \ldots,\left[\begin{array}{ll}\hat{\mathbf{n}}_{H} & d_{H}\end{array}\right]^{T}\right)$

Define $C H_{n e w}(C)$ as the convex hull of the unit wrench extremes of $C$ :

$$
C H_{n e w}(C)=\text { ConvexHull }\left\{\hat{\mathbf{w}}_{1}\left(c_{1}\right), \ldots, \hat{\mathbf{w}}_{L}\left(c_{N}\right)\right\}
$$

Let $C H_{e p s}(G, \epsilon)$ be the intersection of all halfspaces having normals $\hat{\mathbf{n}}_{h}$ and distances $\epsilon_{h}$ :

$$
C H_{e p s}(G, \epsilon)=\left\{\left[\begin{array}{ll}
\hat{\mathbf{n}}_{1} & \epsilon_{1}
\end{array}\right]^{T}, \ldots,\left[\begin{array}{ll}
\hat{\mathbf{n}}_{H} & \epsilon_{H}
\end{array}\right]^{T}\right\}
$$

Then if grasp $C$ is in grasp family $W(G, \epsilon), C H_{n e w}(C)$ contains $C H_{\text {eps }}(G, \epsilon)$ :

$$
C \in W(G, \epsilon) \longrightarrow C H_{n e w}(C) \supseteq C H_{\text {eps }}(G, \epsilon)
$$

This Proposition describes the volume $C H_{e p s}(G, \epsilon)$ in $\mathbb{R}^{6}$ (possibly empty) that is contained within the convex hull of contact wrenches of any grasp in the family $W(G, \epsilon)$. This volume is shown for a $2 \mathrm{D}$ example in Figure $4 \mathrm{C}$.

\subsection{Meeting Design Goals}

Because $C H_{e p s}$ in Equation 11 is determined by the values of $\epsilon_{h}$, properties of grasps in $W(G, \epsilon)$ can be controlled through careful selection of these parameters.

\section{Force Closure}

Grasps in $W$ will be force-closure if the convex hull of the contact wrenches available from any grasp in $W$ contains the origin in its interior. By Proposition 1, it is sufficient that $\mathrm{CH}_{\text {eps }}$ in Equation 11 contain the origin in its interior. This goal is achieved by setting the following constraint:

$$
\epsilon_{h}>0 \quad h=1, \ldots, H
$$

Any small number can be used for all $\epsilon_{h}$ to ensure that force closure is possible for all grasps in $W(G, \epsilon)$.

\section{Grasp Quality Threshold for a Given Task}

A force-closure grasp is not necessarily a desirable grasp, as it may result in large internal forces to counter small external wrenches. A more useful objective may be to synthesize a family of grasps which exceed a given grasp quality threshold. We define grasp quality as follows: 
Definition 1. Grasp quality is the reciprocal of the sum of magnitudes of contact normal forces required to achieve the worst case wrench in a task set.

This definition of grasp quality is based on the notion that the "effort" required for a grasp is related to the sum of magnitudes of contact normal forces as expressed, for example, in [8], [10], and [30].

Now, suppose we are given a task $T$ described as the convex hull of $K$ points $\mathbf{s}_{k}: T=$ ConvexHull $\left(\mathbf{s}_{1}, \ldots, \mathbf{s}_{K}\right)$. For this task, the quality of the example grasp can be defined as follows (e.g., [31]):

$$
Q(G)=\min _{h=1}^{H}\left(\frac{d_{h}}{\max \left(\mathbf{0}, \max _{k=1}^{K}\left(\mathbf{s}_{k} \cdot \hat{\mathbf{n}}_{h}\right)\right)}\right)
$$

To obtain new grasps with quality at least $\beta Q(G)$, set $\epsilon_{h}$ as follows:

$$
\epsilon_{h}=\beta Q(G) \max \left(\mathbf{0}, \max _{k=1}^{K}\left(\mathbf{s}_{k} \cdot \hat{\mathbf{n}}_{h}\right)\right) \quad h=1, \ldots, H
$$

Using Proposition 1, we can show easily that $C \in W(G, \epsilon)$ and Equation 15 imply that

$$
Q(C) \geq \beta Q(G)
$$

\section{Grasp Quality Threshold When No Task is Given}

Now suppose the task is not known. We note that Equations 14 and 15 imply that $\epsilon_{h} \leq \beta d_{h}$ for any task $T$. As a result, we can also obtain grasps with quality at least $\beta Q(G)$ by setting

$$
\epsilon_{h}=\beta d_{h} \quad h=1, \ldots, H
$$

Equation 17 is intriguing because it shows that contact forces can be bounded relative to an example without measuring those forces and without knowing anything about the task! However, using Equation 15 when the task is known may result in larger contact target regions corresponding to directions where the example grasp is unnecessarily strong.

\subsection{Computing Contact Regions}

Grasp family $W(G, \epsilon)$ (Equation 4) is expressed in a manner independent of object geometry. The equation

$$
W(G, \epsilon)=\left\{c_{1}, \ldots, c_{N}: c_{i} \in W_{n}, \quad n=1, \ldots, N\right\}
$$

describes all possible combinations of contacts that can be generated from a given example using our construction technique. 
The simplest way to filter this set of grasps through an object's geometry is to sample the object surface and test contact points for inclusion in $W_{n}$. Given contact $c_{n}$, and with reference to Equation 6, the problem of determining whether $c_{n} \in W_{n}$ can be specified as follows:

find $L x 1$ vector $\alpha_{l}$ and parameter $\nu_{n, l}$ to maximize $\nu_{n, l}$ such that (19)

$$
\begin{gathered}
\left(Y\left(c_{n}\right) \alpha_{l}\right) \cdot \hat{\mathbf{n}}_{h} \geq \epsilon_{h} \nu_{n, l} \quad \forall h \in \rho_{n, l} \\
\alpha_{l} \geq \mathbf{0} \\
\left\|\alpha_{l}\right\|_{L_{1}}=1
\end{gathered}
$$

Then from Equation 5:

$$
c_{n} \in W_{n} \Longleftrightarrow\left(\min _{l=1}^{L} \nu_{n, l}\right) \geq 1
$$

This problem description states that there must be some unit contact wrench available at $c_{n}$ (i.e., some valid value for $\alpha_{l}$ ) such that all halfspace constraints are met or exceeded by this wrench (i.e., $\nu_{n, l} \geq 1$ for all $l=1, \ldots L$ ).

In the frictionless case, $c_{n} \in W_{n}$ can be determined more easily. From Equation 8 and Figure 4:

$$
c_{n} \in W_{n} \Longleftrightarrow \hat{\mathbf{w}}\left(c_{n}\right) \cdot \mathbf{n}_{h} \geq \epsilon_{h} \quad \forall h \in \rho_{n}
$$

In this case, at each sample point on the frictionless surface the contact wrench $\hat{\mathbf{w}}\left(c_{n}\right)$ is formed and tested for inclusion in $W_{n}$ by checking just a few $6 \mathrm{D}$ dot products.

\section{Reducing Asymmetries (A First Try): Wrench Alignment}

Referring back to Figure 3, one feature of this technique is that properties of the example grasp are retained. In some circumstances, such as that shown in the figure, some of these properties may be incidental and not especially desirable.

Figure 5 shows the difficulty. Figure $5 \mathrm{~A}$ shows the $\tau=0$ slice of the 3D force / torque space for the example in Figure 3(Left). The intersection of $\mathrm{CH}_{\text {orig }}$ (Equation 2) with the $\tau=0$ plane is shown. Compare this to Figure $5 \mathrm{~B}$, constructed from the more nicely aligned example in Figure 3(Right). Figures $5 \mathrm{C}$ and $\mathrm{D}$ show the differences after convex hull $\mathrm{CH}_{\text {eps }}$ (Equation 11) is formed using $\epsilon_{h}$ from Equation 15, with task wrenches in the $f_{y}$ and $\tau$ directions $\left(\left[f_{x}, f_{y}, \tau\right]^{T}=[0, \pm 1, \pm 0.3]^{T}\right)$. The symmetric example from Figure 3(Right) aligns much more closely to the set of forces and torques required 

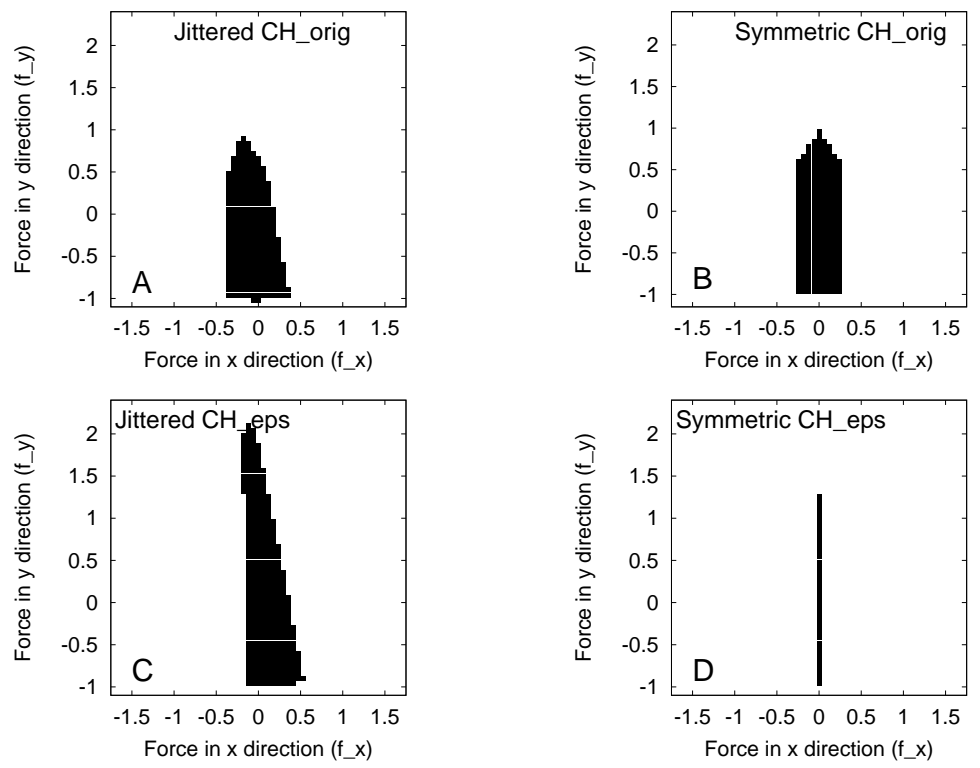

Fig. 5. (A) Interior of $\mathrm{CH}_{\text {orig }}$ for the asymmetrical example from Figure 3(Left). The $\tau=0$ plane is shown in all examples. (B) Interior of $C H_{\text {orig }}$ for the symmetrical example in Figure 3(Right). (C) $\mathrm{CH}_{\text {eps }}$ for the asymmetrical example. Task wrenches are in the $f_{x}=0$ plane. Because the halfspaces of the convex hull are not well aligned with the task wrenches, the volume contained within $\mathrm{CH}_{\text {eps }}$ is large. (D) $\mathrm{CH}_{\text {eps }}$ for the symmetrical example bounds a small volume, which will in general result in larger contact regions on the object surface.

for this task. As a result, given our constructive technique for grasp synthesis, the contact regions in Figure 3(Right) are larger and more symmetrical.

It is also possible to see this phenomenon by examining Figure $4 \mathrm{C}$. With reference to this figure, a poor alignment of halfspaces to task wrenches may produce $\mathrm{CH}_{\text {eps }}$ with large interior and result in small regions $\hat{\mathbf{w}}\left(c_{i}\right)$, while a good alignment may produce smaller $C H_{\text {eps }}$ and tend toward larger $\hat{\mathbf{w}}\left(c_{i}\right)$.

One solution to this problem is to adjust the frictionless contact wrenches of the example grasp so that they are aligned, where appropriate, with the task space or its nullspace. The assumption we are making is that if a wrench is nearly aligned with one of these spaces, then differences from exact alignment are incidental and do not reflect an important property of the example grasp.

Specifically, given contact $c_{i}$, the frictionless wrench at that contact can be expressed as:

$$
\underline{\hat{w}}_{0}\left(c_{i}\right)=\left[\begin{array}{c}
\hat{f}\left(c_{i}\right) \\
\underline{\lambda \hat{\tau}}\left(c_{i}\right)
\end{array}\right]
$$


where $\lambda$ converts from units of torque to units of force. Parameter $\lambda$ can be set to the reciprocal of the radius of gyration of the grasped object, for example. Note that this is not a frame independent representation, and $\underline{\hat{w}}_{0}\left(c_{i}\right)$ should be expressed in the same frame as the task wrenches, typically with origin at the center of mass of the object.

Now divide $\mathbb{R}^{6}$ ( $\mathbb{R}^{3}$ in $2 \mathrm{D}$ ) into two disjoint spaces that together span all of $\mathbb{R}^{6}$ (resp. $\mathbb{R}^{3}$ ). These will be the task space $T$, represented in matrix form with basis directions along the rows, and its nullspace $N$, represented in matrix form with basis directions along the rows. We can project $\underline{\hat{w}}_{0}\left(c_{i}\right)$ into both of these spaces:

$$
\begin{aligned}
\underline{\hat{w}}_{T}\left(c_{i}\right) & =T^{T} T \underline{\hat{w}}_{0}\left(c_{i}\right) \\
\underline{\hat{w}}_{N}\left(c_{i}\right) & =N^{T} N \underline{\hat{w}}_{0}\left(c_{i}\right)
\end{aligned}
$$

Then the aligned frictionless contact wrench is set as follows:

$$
\underline{\hat{w}}_{0}^{\prime}\left(c_{i}\right)=\left\{\begin{array}{l}
\underline{\hat{w}}_{T}\left(c_{i}\right) \text { if }\left\|\hat{\underline{w}}_{T}\left(c_{i}\right)\right\|>a\left\|\hat{\hat{w}}_{0}\left(c_{i}\right)\right\| \\
\underline{\hat{w}}_{N}\left(c_{i}\right) \text { if }\left\|\underline{\hat{w}}_{N}\left(c_{i}\right)\right\|>a\left\|\underline{\hat{w}}_{0}\left(c_{i}\right)\right\| \\
\underline{\hat{w}}_{0}\left(c_{i}\right) \text { otherwise }
\end{array}\right\}
$$

where $a$ is a threshold that determines when a wrench is assumed to be aligned with either the task space or the nullspace and wrench magnitude $\|$.$\| is$ computed using the $L_{2}$ metric in $\mathbb{R}^{6}$. Note that only the fricionless contact wrench is adjusted. The contact model with friction is then developed based on $\underline{\hat{w}}_{0}^{\prime}\left(c_{i}\right)$ instead of $\underline{\hat{w}}_{0}\left(c_{i}\right)$.

Performing alignment in this way fixes the problem shown in Figure 3 for this example (see Figure 6).

\section{Improving the Grasp More Generally: Volume Alignment}

The intuition behind the simple alignment approach just described is to produce larger contact regions by creating a better arrangement of example wrenches $\hat{\mathbf{w}}\left(c_{i}\right)$ with respect to task wrenches $s_{k}$. In terms of the geometry we use to construct contact regions, the goal is to create a $\mathrm{CH}_{\text {eps }}$ that is a closer fit to the task wrench set (Figure 5). Aligning individual contact wrenches can result in halfspace normals that are aligned with the task space and its nullspace.

However, this simple idea does not work well in more complex situations. In this section, we consider the 5 contact example shown in Figure 7 . The task in this case is to tip the object 90 degrees from vertical to a "handle-up" configuration. In this example, results from simply aligning the frictionless contact wrenches with the taskspace or nullspace are significantly worse than the original example.

There are at least two problems. First, unlike the example in Figure 3, the object itself is not symmetric. If we are targetting a specific object geometry, 


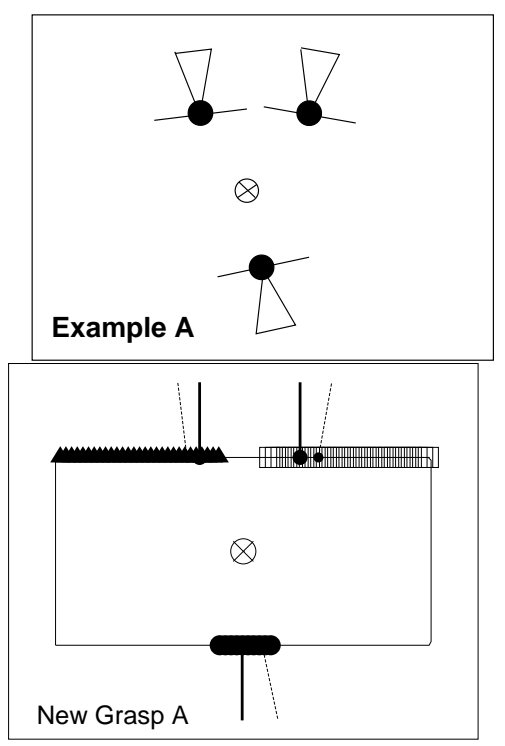

Fig. 6. Frictionless contact wrenches from the asymmetric example can be aligned with the task space or its nullspace to improve the size and balance of contact regions in this example. In the bottom figure, the dotted lines are frictionless contact wrenches before alignment, and the solid lines are frictionless contact wrenches after alignment.

that geometry should be taken into account. Second, the wrench alignment algorithm does not capture the geometry of the task wrench space, only its principal directions. This example of tipping the object onto its side involves both an essentially 2D task space and a limited (90 degree) range of forces within that $2 \mathrm{D}$ space.

To produce better results, we need to more directly address the goal of producing a good fit of $C H_{e p s}$ to the task set $s_{k}$. One possible algorithm, that locally tunes an example to a specific object geometry is as follows:

Iteratively adjust contact positions on the surface of the object to reduce the (six-dimensional) volume of $\mathrm{CH}_{\text {eps }}$.

Figure 7 shows results of adjusting contacts to achieve this goal while keeping them within a specified distance of the original contact locations and normals. Overall, more flexibility is available in the adjusted example than in the original. 

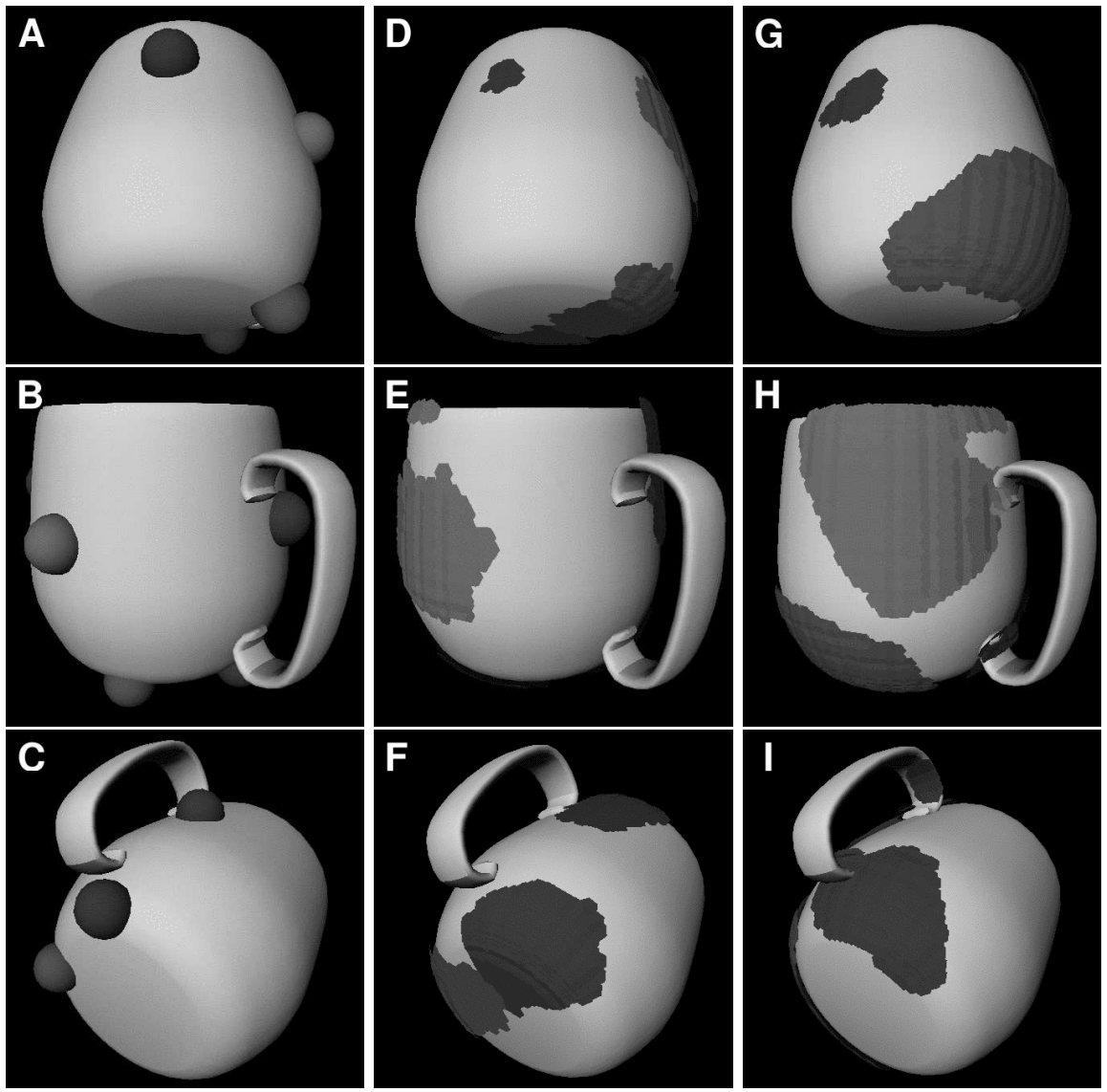

Fig. 7. (A,B,C) Example 5-contact grasp. The task is to tip the object 90 degrees from a vertical to "handle up" configuration. The contact model is hard finger contact with friction, and the coefficient of friction is 1.0. (D,E,F) Contact regions obtained from this example with no adjustment. Grasps in this family are at least $36 \%$ as good as the example grasp. (G,H,I) Contact regions after adjustment. The quality threshold is the same as in figures D through F. Note that the top contact in figures $\mathrm{C}, \mathrm{F}$, and I has moved to the underside of the handle in the optimized example. 


\section{Conclusions}

Human grasp examples can be a rich resource, illustrating sophisticated and successful grasping strategies. To make use of these examples, it must be possible to adapt them to new situations, and we have shown that this can be done in a way that provides some flexibility and also provides guarantees on closure and quality properties of the results.

However, examples have the flaw that they were not necessarily engineered for general use; they are in a sense "found artifacts." As such, any one example is unlikely to be perfect for a given task and class of objects.

We are very interested in how one might develop an optimal example or set of examples. In this paper, we have begun to explore how a given example might be tuned to a task and / or object geometry. However, ultimately an optimal set of examples should take into account all of the constraints of hand structural and functional properties, object geometries for a class of objects, and also the intended task.

Some of our near term research goals are to better understand the dimensionality of human grasps observed in manipulation of everyday objects such as tools and to better understand the key collections of contact models that might represent these grasps. In the longer term, perhaps a next generation taxonomy can be developed along the lines of that of Cutkosky and Howe [5], for example, but which also exploits measurement and analysis technology available today and contains results that can be applied with bounds on applicability and guarantees on the quality of the resulting grasps.

\section{Acknowledgments}

This research was supported in part by NSF CAREER award CCF-0343161.

\section{References}

1. S. Akella and M. T. Mason. Posing polygonal objects in the plane by pushing. International Journal of Robotics Research, 17(1):70-88, January 1998.

2. A. Bicchi. On the closure properties of robotic grasping. International Journal of Robotics Research, 14(4):319-334, 1995.

3. A. Bicchi. Hands for dexterous manipulation and robust grasping: A difficult road toward simplicity. IEEE Transactions on Robotics and Automation, 16(6):652-662, 2000.

4. I-M. Chen and J. W. Burdick. Finding antipodal point grasps on irregularly shaped objects. IEEE Transactions on Robotics and Automation, 9(4):507-512, 1993.

5. M. R. Cutkosky and R. D. Howe. Human grasp choice and robot grasp analysis. In S.T. Venkataraman and T. Iberall, editors, Dextrous Robot Hands. SpringerVerlag, New York, 1990. 
6. B. R. Donald, J. Jennings, and D. Rus. Information invariants for distributed manipulation. International Journal of Robotics Research, 16(5):673-702, 1997.

7. M. Erdmann. An exploration of nonprehensile two-palm manipulation. International Journal of Robotics Research, 17(5), 1998.

8. D. G. Kirkpatrick, B. Mishra, and C. K. Yap. Quantitative Steinitz's theorems with applications to multifingered grasping. In Proc. 20th ACM Symposium on Theory of Computing, May 1990.

9. Y. Li, Y. Yu, and S. Tsujio. An analytical grasp planning on given object with multifingered hand. In Proc. IEEE Intl. Conference on Robotics and Automation, 2002.

10. Z. Li and S. Sastry. Optimal grasping by multifingered robot hands. In Proc. IEEE Intl. Conference on Robotics and Automation, 1987.

11. Q. Lin, J. W. Burdick, and E. Rimon. A stiffness-based quality measure for compliant grasps and fixtures. IEEE Transactions on Robotics and Automation, 16(6):675-688, 2000.

12. Y.-H. Liu. Computing n-finger form-closure grasps on polygonal objects. International Journal of Robotics Research, 19(2):149-158, February 2000.

13. K. M. Lynch and M. T. Mason. Dynamic nonprehensile manipulation: Controllability, planning, and experiments. International Journal of Robotics Research, 18(1):64-92, January 1999.

14. G. Mantriota. Communication on optimal grip points for contact stability. International Journal of Robotics Research, 18(5):502-513, May 1999.

15. X. Markenscoff and C. H. Papadimitriou. Optimum grip of a polygon. International Journal of Robotics Research, 8(2):17-29, April 1989.

16. M. T. Mason. Mechanics and planning of manipulator pushing operations. International Journal of Robotics Research, 5(3):53-71, 1986.

17. B. Mirtich and J. Canny. Easily computable optimum grasps in 2-d and 3-d. In Proc. IEEE Intl. Conference on Robotics and Automation, 1994.

18. B. Mishra, J. T. Schwartz, and M. Sharir. On the existence and synthesis of multifinger positive grips. Algorithmica, 2(4):541-558, November 1987.

19. V. Nguyen. Constructing force-closure grasps. International Journal of Robotics Research, 7(3):3-16, June 1988.

20. M. A. Peshkin and A. C. Sanderson. Planning robotic manipulation strategies for workpieces that slide. International Journal of Robotics and Automation, 4(5):524-531, 1988.

21. Nancy S. Pollard. Closure and quality equivalence for efficient grasp synthesis. International Journal of Robotics Research, (in press), 2004.

22. J. Ponce and B. Faverjon. On computing three-finger force-closure grasps of polygonal objects. IEEE Transactions on Robotics and Automation, 11(6):868881, 1995.

23. J. Ponce, D. Stam, and B. Faverjon. On computing force-closure grasps of curved two-dimensional objects. International Journal of Robotics Research, 12(3):263-273, June 1993.

24. J. Ponce, S. Sullivan, A. Sudsang, J.-D. Boissonnat, and J.-P. Merlet. On computing four-finger equilibrium and force-closure grasps of polyhedral objects. International Journal of Robotics Research, 16(1):11-35, February 1997.

25. E. Rimon and J. W. Burdick. Mobility of bodies in contact-Part I: A 2nd-order mobility index for multiple-finger grasps. IEEE Transactions on Robotics and Automation, 14(5):696-708, 1998. 
26. E. Rimon and J. W. Burdick. Mobility of bodies in contact-Part II: How forces are generated by curvature effects. IEEE Transactions on Robotics and Automation, 14(5):709-717, 1998.

27. J. C. Trinkle and R. P. Paul. Planning for dexterous manipulation with sliding contacts. International Journal of Robotics Research, 9(3):24-48, June 1990.

28. A. F. van der Stappen, C. Wentink, and M. H. Overmars. Computing immobilizing grasps of polygonal parts. International Journal of Robotics Research, 19(5):467-479, May 2000.

29. M. T. Zhang and K. Goldberg. Gripper point contacts for part alignment. IEEE Transactions on Robotics and Automation, 18(6):902-910, 2002.

30. X. Zhu, H. Ding, and H. Li. A quantitative measure for multi-fingered grasps. In Proc. IEEE/ASME Intl. Conference on Advanced Intelligent Mechatronics, 2001.

31. X. Zhu and J. Wang. Synthesis of force-closure grasps on 3-D objects based on the Q distance. IEEE Transactions on Robotics and Automation, 19(4):669-679, 2003. 